anthropology \& materialism

\section{Anthropology \& Materialism}

A Journal of Social Research

$3 \mid 2016$

Utopia: The Elsewhere and The Otherwise

\title{
Le récit utopique. Droit naturel et roman de l'Etat
}

The Utopian Narrative: Natural Right and Novel of State. Chapter II: " Heritages or Demarcations?"

El relato utópico. Derecho natural y novela del Estado. Capítulo II: « ¿Herencias o delimitaciones?»

\section{Pierre-François Moreau}

\section{(2) OpenEdition}

Journals

Electronic version

URL: http://journals.openedition.org/am/640

DOI: $10.4000 / a m .640$

ISSN: 2364-0480

Publisher:

CETCOPRA, CRASSH - Center for Research in the Arts Social Sciences and Humanities, Fakultät

Gestaltung - Universität der Künste Berlin

\section{Electronic reference}

Pierre-François Moreau, «Le récit utopique. Droit naturel et roman de l'Etat », Anthropology \&

Materialism [Online], 3 | 2016, Online since 02 December 2016, connection on 19 April 2019. URL :

http://journals.openedition.org/am/640; DOI : 10.4000/am.640

This text was automatically generated on 19 avril 2019.

Tous droits réservés 


\title{
Le récit utopique. Droit naturel et roman de l'Etat
}

\author{
The Utopian Narrative: Natural Right and Novel of State. Chapter II: « Heritages \\ or Demarcations?» \\ El relato utópico. Derecho natural y novela del Estado. Capítulo II: « ¿Herencias o \\ delimitaciones?»
}

Pierre-François Moreau

\section{EDITOR'S NOTE}

Nous avons tenu à republier le chapitre II du livre de Pierre-François Moreau, Le récit utopique. Droit naturel et roman de l'État (Paris, Puf, 1982, p. 25-42), dans le cadre de ce numéro thématique. Indisponible depuis quelques temps, cet ouvrage est pourtant une contribution de tout premier ordre à l'analyse de l'utopie. Son objectif est de cerner le genre utopique, en soulignant ce qui le distingue de genres littéraires proches, comme les voyages imaginaires, mais aussi de courants philosophiques (platonisme) ou religieux (millénarisme) auxquels on l'a souvent associé. Ce travail de caractérisation est mené sur les utopies classiques, telles qu'elles s'écrivent entre l'ouvrage fondateur de Thomas More en 1516 et le tournant opéré à la fin du XVIII siècle, par exemple avec l'An 2440 de LouisSébastien Mercier, lorsque le genre délaisse les projections spatiales en faveur des projections temporelles, pour ne plus dépeindre un ailleurs mais un plus tard. Dans le premier chapitre de son ouvrage, Moreau soutient que le genre utopique entremêle trois types de discours. On y trouve tout d'abord un discours critique sur l'ici et le maintenant, discours qui a pour particularité de ne pas simplement nommer des maux ou des « abus » mais de remonter à leur racine. Ainsi se comprend la condamnation de la propriété privée, que les utopistes ne rejettent pas in abstracto, mais en référence aux multiples fléaux (inégalité des fortunes, avidité des uns, servitude des autres) dont elle est pour eux la cause fondamentale. Un deuxième type de discours est descriptif. Il s'agit de raconter la vie en utopie et de rendre apparents les rouages de la mécanique sociale qui 
s'y déploie. L'utopie ne propose donc pas une constitution idéale, pas plus qu'elle n'éclaire la transition d'une société vers une autre. Elle donne d'emblée la société utopique comme réalisée et entreprend dès lors uniquement $d$ 'en décrire, souvent avec un certain luxe de détails, le fonctionnement (en ce sens, les auteurs d'utopie font de la philosophie politique mais de manière non philosophique). Le récit utopique se donne enfin comme un discours justificatif, dans la mesure où il expose aussi - de manière plus ou moins explicite en fonction des œuvres - les conditions de possibilité de la société qu'il dépeint à ses lecteurs. Il est en d'autres termes inséparable d'une anthropologie, laquelle affermit les descriptions fournies et garantit que celles-ci ne sont pas de simples divagations sans fondement ni cohérence.

Ces quelques précisions permettent de mieux comprendre les enjeux du chapitre 2, " Héritages ou démarcations? », que nous republions ici. Il a pour objectif de dessiner les frontières qui séparent le genre utopique de structures narratives ou conceptuelles voisines : la République de Platon, le mythe de l'Âge d'Or, le messianisme juif, le millénarisme chrétien et l'Autre Monde celtique. Moreau reconnaît l'influence de ces « antécédents » sur les utopies classiques, mais il montre que celles-ci s'en distinguent sur des points très importants. Par un jeu de contrastes, il éclaire donc la singularité du genre apparu avec l'ouvrage de Thomas More, et réaffirme certaines différences sur lesquelles certains passent parfois un peu légèrement.

Avant de laisser place au texte, on peut brièvement essayer de mettre en regard ces thèses avec d'autres contributions décisives sur l'utopie, comme celle de Karl Mannheim. Ainsi que l'a remarqué Pierre Macherey ${ }^{1}$, les approches des deux auteurs sont par bien des aspects diamétralement opposées. L'analyse de Moreau est structurale, construite autour de la notion de genre utopique. L'approche de Mannheim est historique, bâtie en référence à l'idée de conscience utopique. Alors que Moreau s'efforce de circonscrire précisément le périmètre de l'utopie comme genre, ainsi que sa portée (accompagner la naissance de l'État moderne), Mannheim y voit une attitude face au monde radicalement anti-conservatrice, qui a pour conséquence la transformation effective de la réalité sociale. Le critère qui permet au sociologue allemand de juger de ce qui est ou n'est pas utopie est en effet celui de sa mise en œuvre, critère qui ne peut être appliqué qu'a posteriori. Ont par conséquent valeur d'utopie toutes les représentations qui « furent grosses d'effets sur l'être socio-historique $»^{2}$.

En cohérence avec cette approche, Mannheim situe l'origine de l'utopie non dans l'ailleurs fictionnel décrit par More(au), mais dans la violence millénariste de Thomas Münzer. Alors que le millénarisme est - comme le rappelle à juste titre Moreau dans ce chapitre 2 - extérieur au genre utopique, Mannheim y voit l'une des manifestations les plus spectaculaires de la conscience utopique et de sa fonction de " dislocation de l'être ». En revanche, le sociologue allemand ne considère pas que les « mythes, contes, promesses religieuses d'un au-delà, imaginations humanistes » ou « récits de voyage $»^{3}$ soient à proprement parler des utopies. C'est là en quelque sorte un point d'accord avec Moreau, mais l'on voit bien que les raisonnements qui y conduisent les deux auteurs sont bien différents. Si les mythes ne sont pas des utopies, c'est pour Moreau parce qu'ils ne possèdent pas toutes les caractéristiques du genre fondé par More, et qui est demeuré remarquablement stable durant deux siècles et demi. Pour Mannheim, les mythes ne sont pas des utopies parce qu'ils n'ont pas véritablement changé le monde où ils sont apparus, mais se sont contentés d'y ajouter quelques couleurs complémentaires. Ainsi, même s'ils sont d'accord pour séparer le mythe et l'utopie, l'approche théorico-littéraire de Moreau s'oppose à la réflexion sociale-historique de Mannheim. 
On voit par là que si les deux auteurs emploient le même terme (" utopie »), ils ne parlent pas nécessairement de la même chose. Ce n'est qu'un nouvel exemple de la difficulté à saisir une notion que Moreau circonscrit, peut-être de manière un peu trop limitative, à un genre littéraire et à une époque historique. Cela n'enlève rien au fait que son ouvrage, passionnant et limpide de bout en bout, mérite amplement d'être relu et discuté.

\section{I. [La République de Platon $]^{4}$}

1 Si les traits qui définissent comme genre le récit utopique correspondent à une certaine structure intellectuelle, ils se laisseront mieux saisir dans l'approche des frontières qui les séparent des structures voisines. Le rappel des " antécédents » ou des " précurseurs » est un passage obligé des analyses de l'utopie (voire du discours des utopistes euxmêmes). Toute la question reste alors de savoir si on évoque ces antécédents pour approcher mieux, par la comparaison, ce que chacun a de spécifique, ou si on noie chaque exemple dans une aspiration éternelle (et continue) de l'âme humaine à l'ailleurs et au meilleur. Trancher ce problème ne supprimera d'ailleurs pas toutes les questions : car si on opte pour la discontinuité, il faudra alors expliquer comment le récit utopique peut réorganiser des matériaux produits antérieurement, sous la juridiction de genres différents.

2 La difficulté est assez exemplaire avec la République de Platon : on s'applique à y retrouver «la plupart des thèmes que développeront les utopies ultérieures », en particulier «la tendance de l'esprit utopique au dirigisme absolu, la foi dans les règlements stricts, les répartitions précises, une sorte d'ivresse de législation géométrique ${ }^{5} »$. En procédant ainsi, on ne fait que suivre un exemple donné par les utopistes eux-mêmes: Thomas More en effet se réclame de Platon, qui refusa de donner des lois à ceux qui n'accepteraient pas un partage égal de tous les biens; « Ce grand penseur prévoyait avec sagacité que le seul moyen de sauver un peuple c'est l'égalité des conditions ${ }^{6}$ ». Campanella semble même plus fidèle encore à ce "créateur du genre ${ }^{7}$ » en allant jusqu'à la communauté des femmes. Et il est effectivement exact qu'on retrouve un certain nombre de thèmes communs dans les deux types d'univers. Mais en faisant de ces thèmes la marque d'une continuité simple, décèle-t-on l'unité réelle d'un genre bien défini ou ne fait-on que reproduire l'illusion interprétative des utopistes eux-mêmes?

3 La République n'est pas un récit, et elle ne décrit pas le tableau d'une société fonctionnant actuellement (même si c'est imaginairement). Or ce n'est pas là une question de formulation : cette mise en œuvre est nécessaire à l'essence de l'utopie, qui n'est pas un programme et à qui seul le schéma narratif permet de donner vie et présence au monde qu'elle instaure. La République, au contraire, suit la construction habituelle du dialogue platonicien ${ }^{8}$, et elle prend place dans la réflexion grecque sur les différents types de constitution'. Il y est expliqué que les quatre formes principales de gouvernement (timiocratie, oligarchie, démocratie, tyrannie) se succèdent par corruption d'un idéal conforme à la justice. Cet idéal (qui pas un instant n'est supposé réalisé) va donc être reconstruit à partir de cette idée.

4 Le problème de la justice est en effet chez Platon, sinon le problème politique par excellence, du moins le problème qui détermine la politique et permet de la penser (ce qui est dit dès l'Alcibiade : elle est l'art de savoir « ce qu'il vaut mieux faire »). La justice est pensée comme harmonie, et l'établissement de cette harmonie pose les mêmes questions 
à l'égard de l'individu et de l'état. Alcibiade déclare en effet : «l'homme c'est l'âme ${ }^{10}$ » et c'est la tripartition de l'âme qui rend possible l'analogie entre individu et Cité : les différentes classes qui se partagent la Cité correspondent aux différentes parties de l'âme. La même harmonie qui règne entre nous, thumos et epithumia doit régner entre gouvernants, guerriers et artisans, chacun ayant sa vertu propre : la sagesse, le courage, la tempérance. Dans une telle hiérarchie, chacun a sa place et la justice consiste précisément à ce que chacun remplisse sa fonction. Il s'agit d'assurer « ce concert, cet accord naturel de la partie inférieure et de la partie supérieure pour décider laquelle des deux doit commander dans l'État et dans l'individu ${ }^{11} »$.

Il est clair alors que la politique de Platon se fonde sur sa doctrine de l'âme, et que celle-ci étant pluraliste et hiérarchiste, sa politique l'est aussi. C'est pourquoi les conditions des citoyens sont non pas égales comme le dit More, mais dans un rapport bien proportionné, ce qui est tout différent. On aurait tort, par ailleurs, d'interpréter immédiatement sa République en termes d'égalité ou d'inégalité sociale, car cette notion de la justice n'appartient pas d'abord à un registre « social »; mais ceux qui réfléchissent à la politique à l'intérieur d'une atmosphère où l'État est devenu une machine à gérer la société (et c'est le cas des utopistes classiques) ne peuvent prendre en vue les problèmes éthiques ${ }^{12}$ que pose Platon qu'en les traduisant immédiatement en termes sociaux. C'est même exactement ce par quoi, aux yeux des Grecs, Platon excédait le champ de la Politique (il suffit de penser aux critiques d'Aristote ${ }^{13}$ qui se ramènent au fond à montrer que l'unité de l'État platonicien est celle d'une famille plus que celle d'une Cité) qui le prédispose à être détourné et utilisé par cette nouvelle version de la politique qui prend figure au XVI siècle. Car son insistance à doter l'État d'une unité forte semblera immédiatement lisible pour ceux qui l'objectivent assez pour en faire le personnage d'un récit.

Mais cette possibilité de relecture n'est pas une identité. La conception de la justice comme harmonie divise d'emblée la Cité en ordres, comme la condition première de son existence. Chez More au contraire, lorsqu'on voit apparaître des ordres (à propos des lettrés) ils se réintroduisent comme en fraude ; et c'est la communauté des biens qui est la clef de l'État, parce qu'elle supprime les racines premières d'un mal économique et social. La cité platonicienne ne connaît le communisme que sous le primat d'une division en classes (il n'existe que dans la classe des gouvernants) et il sert essentiellement à résoudre les conflits éthiques entre les hommes - exactement : entre les hommes de la classe supérieure. Il assure donc, en un sens, la perpétuation de la société de classes, que dans l'utopie il sert à supprimer.

On peut alors trier les thèmes qui circulent entre les deux genres : seront communs tous les traits qui, une fois détournés de leur insertion générique, peuvent être réutilisés dans un autre contexte. Encore faut-il que les circonstances donnent les raisons de cette réinsertion : dans le climat de la Renaissance, où Érasme veut voir dans la communauté platonicienne un modèle moral assimilable à celui qui est enseigné par le christianisme, un certain nombre de traits peuvent être pris non plus comme des moyens de réaliser l'idée de Justice, mais comme des mises en œuvre imagées de l'unité politique et sociale de l'espèce humaine. C'est le filtre constitué par cette dernière et par la nouvelle conception de l'État qui laissera passer par exemple le refus du luxe, la prise en charge de l'éducation par la Cité, un certain géométrisme; qui en bloquera d'autres, comme la communauté des femmes : car la plupart des utopistes la refusent pour fonder sur la cellule familiale une forme de contrôle social qui corresponde mieux à leur idéal juridique que les corporations ou d'autres collectivités. On peut expliquer par la même différence la 
présence dans les utopies classiques de traits absents chez Platon, comme le rôle universel du travail ou le désir affiché de réaliser le bonheur de chacun. C'est pourquoi la référence au modèle platonicien n'est ni une garantie d'authenticité, ni un simple contresens : elle est une illusion nécessaire et qui a la rigueur d'une réfraction ${ }^{14}$.

\section{II. [L'Âge d'or]}

8 Il est une autre référence grecque qu'invoquent parfois les écrivains utopiques, et à laquelle on se sent obligé de les rattacher, sans toujours évaluer le prix de ce rattachement. Non plus une œuvre, mais un mythe qui court à travers des générations d'œuvres : l'Âge d'or. Évocation d'une terre sans mal située aux commencements de l'humanité, avant l'histoire, dans un climat semi-divin : on n'y connaissait ni le travail, ni la souffrance, ni la discorde, et la terre donnait d'elle-même des récoltes abondantes. Irrémédiablement perdu, l'âge d'or a été remplacé par des situations de plus en plus dégradées : âges d'argent, d'airain, et finalement de fer, qui est le nôtre - depuis aussi loin que la mémoire s'étende.

9 Le classique de cette nostalgie est Hésiode qui, dans les Travaux et les Jours, évoque ces premiers temps à jamais disparus : «D'or fut la première race des hommes périssables (...) Ils vivaient comme des dieux, le cœur libre de soucis, à l'écart et à l'abri des peines et des misères, la vieillesse misérable sur eux ne pesait pas (...) tous les biens étaient à eux : le sol fécond produisait de lui-même une abondante récolte et eux, dans la joie et la tranquillité, vivaient de leurs champs, au milieu de biens sans nombre ${ }^{15} »$. On remarquera que tous les traits sont du même registre : celui de la nature ; tout semble s'unifier sous le signe de la concorde entre la terre et l'homme, et aussi entre l'homme et le temps : ni effort, ni décrépitude. Quant à la concorde entre les hommes elle va tellement de soi qu'elle n'est même pas évoquée. Hésiode pour décrire l'âge d'or, n'éprouve le besoin de parler ni des guerres, ni des Cités, pas même pour les nier.

10 Le ton change avec Ovide, dont les Métamorphoses vont transmettre le thème (comme tant d'autres) au Moyen-Age latin et à la Renaissance. Chez lui au contraire, l'évocation de l'âge d'or se définit d'abord en termes sociaux et non en termes naturels; il est vrai que c'est négativement, mais tout l'arsenal de la loi et de la justice, qui n'était pas même mentionné chez Hésiode, est appelé dans sa récusation même: "L'âge d'or naquit le premier, qui, sans répression, sans lois, pratiquait de lui-même la bonne foi et la vertu. On ignorait les châtiments et la crainte; des écrits menaçants ne se lisaient point sur le bronze affiché en public ; la foule suppliante ne tremblait point en présence de son juge, un redresseur de torts était inutile à sa sécurité ${ }^{16} »$. La nostalgie d'un monde sûr et tranquille s'ajoute donc au rêve d'une nature féconde; sûr et tranquille non parce qu'il serait bien réglé, mais parce que la spontanéité des hommes saurait encore se passer de règles. A la plénitude de la nature correspond le degré zéro de la sociabilité : on a pu dire que dans ce poème, le mot-clef semble être " nondum » (pas encore ${ }^{17}$ ); pas de trompettes ni de casques, pas encore de fosses autour des cités, ni même de voyages : ni guerre, ni méfiance, ni communication entre les hommes. Et c'est seulement dans un second temps, et comme par analogie, qu'est évoqué l'âge d'or agraire: «la terre aussi, libre de redevances, sans être violée par le hoyau ni être blessée par la charrue, donnait tout d'elle-même ${ }^{18}$ » et le printemps était éternel ${ }^{19}$. C'est à l'âge d'argent qu'apparaissent les saisons, comme les combats à l'âge de bronze et, à l'âge de fer, la fraude, les voyages et la limitation des champs. On remarquera donc que le "communisme», absent dans la 
version hésiodique, n'est mentionné ici que très vaguement et comme « récessivement ${ }^{20}$ ", à propos de sa disparition. Ce qui n'a rien d'étonnant: l'évocation de l'âge d'or ne s'encombre pas de rapports sociaux (si ce n'est pour les rejeter indistinctement), elle ne peut donc avoir de doctrine concernant la propriété des biens. La seule façon de penser à ces derniers est de dire qu'ils abondent, non de s'interroger sur leur répartition.

11 Un monde sans maux naturels et sans contraintes sociales : c'est sous cette double forme que le topos se perpétuera désormais, et les auteurs chrétiens le reprendront à leur compte : ils en feront le témoin d'une époque passée où Dieu était véritablement adorée ${ }^{21}$. On verra donc ce mythe grec de l'origine coexister avec ceux que fournissait la tradition judéo-chrétienne en principe triomphante. Ainsi, "l'Occident a vécu avec deux mythologies côte à côte, dont l'une servait de support à sa théologie et à son éthique, et dont l'autre fournissait aux arts modèles et conventions » note $\mathrm{H}$. Levin, qui ajoute que si la frontière était en principe nettement tracée entre les deux univers, nombreux furent les points d'interpénétration ${ }^{22}$. Dante, au chant XXVIII du Purgatoire assimilera directement Âge d'or et Paradis terrestre :

«Les poètes anciens, qui ont tant célébré

Et l'âge d'or et son heureux état,

Peut-être de ce lieu rêvaient sur le Parnasse

Ici fut innocent le premier couple humain,

Ici règnent les fleurs et les fruits éternels,

Cette eau, c'est le nectar que tous ils ont chantée ${ }^{23}$ ».

12 Autant l'utopie est facile à dater dans son apparition, autant l'âge d'or semble être une constante qui excède toute périodisation: il serait donc tentant de ne voir dans la première qu'une des multiples formes prises par le second; et ce serait d'autant plus facile que maintes fois, le narrateur visitant telle ou telle île à la société parfaite s'écrie qu'il est face à l'âge d'or retrouvé.

Certains caractères pourtant empêchent de les réduire complètement l'un à l'autre :

14 - leur situation à l'égard du temps n'est pas la même. Non seulement l'âge d'or est dans le passé, mais il est irrémédiablement aboli : l'histoire ne se constitue que de sa disparition. À la différence du Paradis terrestre de la tradition judéo-chrétienne, dont l'espérance du salut peut atténuer la perte, le temps originaire du premier âge n'est ici pensable que sur le mode de la nostalgie. La disposition utopique ne s'inscrit nullement dans ce registre élégiaque : elle suppose une comparaison de deux mondes strictement contemporains, et l'État-modèle est précisément tel qu'il ne saurait connaître la dégradation ;

15 - dès que l'on quitte l'évocation vague d'un bonheur parfait, on s'aperçoit que le contenu en est différent. L'utopie est une société autre, alors que l'âge d'or est antérieur à la société. Si la première race humaine est censée avoir été en accord avec la nature et avec elle-même, les mondes utopiques sont au contraire ceux où la société, son État et ses lois sont non pas inexistants, mais organisés de la meilleure façon possible pour combattre la nature et se l'approprier. Rêve du technicien contre rêve du paysan, pourrait-on dire avec quelque à-peu-près. Aucun des problèmes fort précis dont l'utopie se veut position et résolution n'est même évoqué dans le mythe : toutes ces questions de technologie sociale y sont simplement supprimées par avance. Toute la différence est celle qui sépare la spontanéité de la construction. L'attitude à l'égard du travail est indicative : l'âge d'or l'ignore, parce qu'il met entre parenthèses tout ce qui est de l'ordre de la nécessité. Le paradis terrestre de l'utopie est plus proche sur ce point de l'Eden judéo-chrétien : jardin 
admirable, mais que déjà l'homme devait travailler, - et où déjà, peut-être le travail est pensé comme ayant une valeur formatrice ${ }^{24}$ : idée inconcevable dans le cadre de l'âge d'or mais familière a tout utopiste ;

- enfin les deux mondes n'ont pas le même statut dans leur expression : si l'utopie est un genre philosophique et littéraire, l'âge d'or est un motif utilisé partout, mais qui (sauf erreur) ne donne jamais lieu à un ouvrage entier; ce qui est assez compréhensible, son contenu vague et purement négatif se prêtant facilement à l'allusion, mais rendant impossible une élaboration ordonnée d'assez longue haleine. Plus qu'un genre, plus qu'un univers autonome, la nostalgie de la première race des hommes est un matériau qui peut se réinsérer dans des genres ou des univers multiples, au point précis où leur parcours les amène à rêver de spontanéité (même si ce parcours, dans son mouvement d'ensemble, n'est pas fondé sur la spontanéité); et c'est bien le cas avec l'utopie : on ne s'écrie qu'elle est l'âge d'or que lorsqu'on en considère le résultat - alors que l'originalité utopique consiste précisément non dans le résultat mais dans son effort et la systématique de ses moyens. Le narrateur qui constate simplement le bonheur et la concorde des utopiens fait halte un instant dans son parcours descriptif; mais sa description montrera par quelle organisation, quel travail et quelles difficultés vaincues s'obtiennent concorde et bonheur. L'utopie est l'âge d'or retrouvé et reconstruit - ce qui veut dire (puisque celui-ci est à jamais aboli, et qu'il n'est pas susceptible de construction) qu'elle n'est pas l'âge d'or du tout ${ }^{25}$.

\section{III. [Messianisme juif et millénarisme chrétien]}

17 Par deux fois au moins (dans la question du temps et dans celle du travail) c'est par ses aspects communs avec la doctrine du Paradis terrestre juif et chrétien que l'utopie se distingue de l'Âge d'Or. Ce n'est peut-être pas une coöncidence: même à travers ses interprétations hétérodoxes, même après détournements et contresens, la Genèse a marqué la plupart des conceptions du monde qui se sont exprimées en termes d'origine ou de recommencement. On ne saurait donc sous-estimer les liens que peut entretenir le récit utopique avec une autre tentative d'instaurer un monde meilleur : le courant qui, longuement, opiniâtrement, a espéré, face à la corruption du monde actuel, en l'établissement du " royaume de Dieu sur la terre ». Il faut y compter à la fois certaines interprétations du messianisme juif, le millénarisme des premiers chrétiens, les mouvements médiévaux tels que le joachimisme et le renouveau des espérances chiliastiques aux $\mathrm{XVI}^{\mathrm{e}}$ et $\mathrm{XVII}^{\mathrm{e}}$ siècles. Il s'agit là de moments intellectuels assez hétérogènes, mais où il n'est peut-être pas impossible de déceler une attitude à certains égards commune.

Gershom Scholem a montré à quel point le messianisme juif a pris, vers les débuts de notre ère, un aspect nouveau ${ }^{26}$. Alors que le prophétisme ne connaissait qu'un seul monde, au sein duquel se déroulaient tous les événements de l'histoire sacrée, y compris la rédemption finale, la nouvelle eschatologie parle de deux mondes qui se succèdent et se tiennent dans un rapport antithétique. Il s'agit désormais d'autre chose que des fins dernières annoncées par les prophètes: rejet de l'idolâtrie, gloire d'Israël et rétablissement de la maison de David. Des thèmes beaucoup plus radicaux apparaissent : destruction d'un monde qui ne mérite que la destruction, naissance d'un autre sur les ruines du premier, affrontement cosmique de la lumière et des ténèbres. 

fait, dans les premiers temps, de communautés qui attendent une Seconde Venue assez rapide du Christ. Puis, une fois cette version officiellement rejetée par l'Église ${ }^{27}$, il demeure l'espérance de groupes qui spéculent sur la venue d'un règne de l'Esprit, où s'achèvera l'histoire sacrée et où s'instaurera un ordre nouveau. De telles espérances prennent un nouveau souffle et un accent plus exigeant dans le sillage de la Réforme, lorsque certains, débordant Luther, reconnaissent au peuple chrétien entier la possibilité d'être animé par l'Esprit, et le droit alors, de bouleverser les institutions littérales qui corrompent et obscurcissent le message divin; on voit alors se mêler critiques de la hiérarchie ecclésiastique, promesses d'un monde nouveau, et, souvent revendications sociales ou prophétisme politique ${ }^{28}$. orthodoxe selon laquelle le Christ a ouvert la phase finale de l'histoire du Salut. Dans un cas parce qu'il n'est pas reconnu pour Messie, dans l'autre parce qu'on attend encore un troisième règne. Thèse théologique - mais qui a des conséquences terrestres immédiates : ce monde-ci, celui où l'on vit, est (en partie au moins) discrédité. Il devra être supprimé pour que puisse se poursuivre la rédemption de l'humanité guidée par le plan divin. Un autre monde est en marche, dont l'avènement va renverser ce qui dans celui-ci est norme et qui n'est, du point de vue eschatologique, que somme d'iniquités. Une telle attente ouvre sur toute autre chose que le salut individuel de l'âme après la mort : dans l'histoire même (et peut-être ici et maintenant si les temps sont venus) il va se passer quelque chose de dimension divine - quelque chose qui doit, précisément, bouleverser l'histoire ; les signes du monde vont s'inverser ; ce qui était en bas sera en haut; ce qui était interdit sera permis ; une telle attitude fait appel à des éléments communs à diverses situations religieuses (on peut penser à l'antinomisme des anciens gnostiques) mais ici ils débouchent sur une refonte politique du monde - politique et religieuse à la fois. Ce qui donne un visage reconnaissable à tant de doctrines diverses, où la fusion de l'exaltation religieuse et de critiques violentes de la société se fait sous le signe du Troisième Règne ou, sous quelque autre nom, du cataclysme imminent qui va renverser le monde ancien.

On peut bien sûr distinguer entre des variantes modérées ou violentes de cette attente et de cette prédication ${ }^{29}$; mais les unes et les autres ont un point commun: une telle structure mentale, sous des apparences parfois extrémistes, permet une attitude «objectale » à l'égard du monde: celui-ci n'est pas pris comme le réceptacle obligé et neutre des actions humaines, mais il se révèle comme beaucoup plus lié à celles-ci, et à leur nature, qu'il ne paraît (corrompu si elles sont corrompues, destructible si elles sont destructibles). Il devient un objet que l'histoire eschatologique peut écarter. On ne saurait minimiser l'analogie entre une telle conception et le procédé de formation de l'utopie. Par le caractère radical de leur critique de l'ici, les deux visions sont indéniablement apparentées. C'est ce qui explique la présence d'idéologèmes communs, comme les figures du monde à l'envers, ou la communauté des biens (voire des femmes), visiblement conçues comme subversion du monde existant.

Parenté n'est pas identité. Martin Buber, qui se pose la question au début de son livre Utopie et Socialisme, trace deux lignes de démarcation qui cernent assez bien la difficultée ${ }^{30}$. S'achevant sur l'image d'un temps parfait, la contemplation messianique est d'ordre cosmique; alors que, se référant à l'image d'un espace parfait, l'utopie reste «limitée selon son essence au cercle intérieur de la société ». D'autre part l'acte décisif est situé 
dans le premier cas en haut (l'arrivée des derniers temps requiert une intervention divine) et dans le second en bas (le seul facteur en jeu est la volonté humaine consciente).

II faut insister sur le sens de la référence temporelle : le futur eschatologique n'est pas un simple symétrique du passé de l'âge d'or. Car il implique une dimension cosmologique, dans la mesure où il marque le retour de la positivité divine dans une histoire qui l'avait perdue. C'est là tout autre chose que de remarquer que les hommes, ou leurs chefs, ne respectent plus les préceptes de l'évangile, et que de demander qu'ils s'y soumettent de nouveau dans l'intérêt de tous: une telle remarque et un tel vœu forment un lieu commun moral que l'on peut retrouver non seulement dans le discours du narrateur utopique, mais chez les satiriques, les pamphlétaires ou les prédicateurs. Mais l'attente millénariste est espérance d'une réconciliation qui engage l'univers entier, et c'est pourquoi on retrouve si facilement dans son expression les signes surnaturels d'habitude absents de l'utopie. De même, si des deux côtés s'affiche la même tendance à déchiffrer le monde à l'aide d'une clef unique, la clef n'est pas la même : le millénarisme s'appuie sur une apocalypse, c'est-à-dire sur une révélation de ce qui est caché, mais ce qui est caché, ce sont les secrets de la création et du salut; dans le cas de l'utopie, l'explication (qui, même succincte, se donne comme démonstration et non révélation) déplace le problème au seul terrain de l'organisation de la Cité : la propriété, la famille, l'État ; même lorsqu'il est question de religion, il s'agit moins d'en énoncer une que de savoir laquelle - ou lesquelles - l'État doit énoncer, ou tolérer. De la structure générale de l'univers nous ne saurons rien et c'est peut-être cette pauvreté de la raison utopique qui, en un sens, en fait la modernité.

On peut même, dès lors, facilement prévoir à quels moments on verra le millénarisme affleurer au sein du récit utopique : ce sera lorsqu'on aura affaire à une variante dont le dispositif tendra à associer les décisions sociales à des données de dimensions cosmiques. Le cas est flagrant avec l'astrologie chez Campanella.

Il resterait une question à poser : il est difficile de penser la logique du millénium en la dissociant de l'engagement personnel du millénariste; celui-ci attend, espère, provoque dans une certaine mesure les événements dont il annonce l'imminence; il dénonce le monde corrompu et aspire à sa fin; et si cette fin ne vient pas, il se fabrique une conscience de crise prolongée, qui lui permet de camper dans un monde qui n'a pas encore trouvé son abîme, sans s'en juger solidaire et sans démentir son jugement ; il peut ainsi user, pour ses besoins, de ce que cette sphère déjà idéalement frappée lui ménage, sans y associer de valeurs vitales. Celles-ci demeurent établies dans les événements attendus, et dans le groupe qui se soude de les attendre encore. Que fait, quant à lui, le narrateur utopique? Celui qui, à l'intérieur du récit, est chargé de décrire la marche parfaite de l'ailleurs, et de condamner, directement ou par contrecoup, l'ici? Le plus souvent, on ne nous le dit pas. Explicitement, comme Raphaël Hythlodée, il refuse de contribuer à améliorer ce monde-ci, manière insistante d'en souligner les défauts irrémédiables. Au mieux, il s'efface dans son récit ; au pire, il en périt. Au début de La terre australe connue, Gabriel de Foigny nous apprend comment il a obtenu le manuscrit attribué au narrateur : celui-ci descend du vaisseau qui l'amène de Madagascar (dernière étape racontée dans le livre) : "On débarqua avec quelque précipitation comme c'est l'ordinaire après de si longs voyages, ce qui fit que notre Auteur, affaibli sans doute des incommodités que cause un chemin de trois ou quatre mille lieues, passant sur la planche, glissa et tomba dans l'eau avec une petite valise qu'il portait »; la valise contient le récit qu'on lit ensuite, bien sûr, et c'est peut-être un emblème de la situation du narrateur 
utopique que ce récit qui lui survit, lui-même n'ayant qu'à se noyer pour attester que d'un monde à l'autre il n'est pas de passage ${ }^{31}$. Le récit établit la supériorité de l'utopie sur nos régions ; il ne laisse pas de place à une pensée de la transition, fût-elle catastrophique; la fadeur de celui qui raconte, ou l'impasse de son existence, en redoublent la preuve ${ }^{32}$.

\section{IV. [L'Autre Monde celtique]}

26 présent et lié à lui d'une certaine manière: par le biais de la reconstruction, de la dégradation ou de l'espérance. Dans le cas du récit utopique, il s'agit d'éloignement dans l'espace et cet éloignement se réduit et se matérialise par une navigation - et son achèvement catastrophique, égarement ou naufrage. Ce n'est pas un accessoire, puisque c'est ce fait initial qui sert de pivot à la présentation narrative de la Cité de nulle part. C'est en ce point que le genre se rapproche d'un autre de ses « héritages » : l'Autre Monde celtique et les itinéraires qui y conduisent.

Celui-ci « était à la fois le pays des morts, plus exactement de ceux qu'on croit morts, mais qui sont peut-être encore vivants, et celui des dieux, des déesses et des fées ; régions bienheureuses où l'on ne vieillit pas, où le temps s'abolit, où un jour vaut un siècle ${ }^{33}$ ». Cette différence de rythme souligne le décalage des univers : tout ce qui touche à l'au-delà semble ne pas avoir de fin et les passions ou le bonheur y sont marquées du signe de la perpétuité ${ }^{34}$. Enfin, ce monde est lumineux, à la différence du sheol hébreu ou des Enfers homériques. Mais, comme tel, il pourrait n'être qu'une variante supplémentaire des îles Fortunées s'il n'avait un dernier caractère qui le rend éminemment " productif » sur le plan littéraire : la frontière de cet Autre Monde est franchissable.

La limite qui sépare les deux séjours, océan, fleuve dangereux ou mur de brouillard, s'ouvre parfois pour laisser passer le héros. En poursuivant trop loin un cerf de couleur blanche, en franchissant un gué interdit ou en entreprenant une navigation lointaine, un être humain peut être appelé à partager pour un temps la vie des bienheureux. Il devra alors affronter des épreuves énigmatiques, et savoir garder le secret lorsqu'il repassera dans le monde de la vie habituelle. De telles conceptions, issues d'une mythologie préchrétienne, passent assez facilement dans les littératures médiévales. On a souligné en effet à quel point ce thème possédait une valeur structurale : il engendre une bipartition de l'univers qui se prête éminemment au traitement romanesque: "Dans tout récit, conte ou roman où il intervient, deux plans s'établissent aussitôt, celui d'un monde terrestre, banal, vulgaire, quotidien, et celui d'un monde supérieur, idéal, qu'il s'agisse de l'Autre Monde féerique ou de l'Autre Monde courtois, ou des deux a la fois. Dans cette structure, le rôle de l'aventure, de l'événement merveilleux ou singulier, est de permettre le passage du plan inférieur au plan supérieur ${ }^{35} »$. Elle permet de faire basculer le récit vers ce que Kubin appellera l'Autre Côté. C'est ce thème, et ce qu'il implique, qui permet peut-être de penser l'unité d'un genre comme le lai. Mais c'est à une autre de ses résonances qu'il faut ici penser: la navigation qui fait passer d'un monde à l'autre quelqu'un qui en reviendra édifié - c'est, dans le principe, sinon dans ce qu'on découvre en ce monde, une assez bonne définition de l'ouverture de chaque utopie. Certes, le côté politique et social de celle-ci a été suffisamment souligné pour qu'on ne s'attarde pas à identifier les contenus; en revanche, le procédé formel est effectivement analogue, en particulier en ce qu'il n'est pas purement formel : l'acte décrit de la navigation est la mise 
en œuvre d'une idée profondément ressentie - le caractère incommensurable des deux univers, celui que nous connaissons et celui qui se laisse découvrir à l'issue d'un voyage presque impossible. L'analogie est surtout claire si l'on songe non pas à des exemples littéraires, mais à des Itinéraires christianisés, comme le voyage de Saint-Brendan : celuici emmène ses moines pour leur montrer l'au-delà (référence chrétienne, mais le mode d'approche demeure le même) et ils reviennent convaincus par ce qu'ils ont vu.

Ici encore pourrait être posée la même question qu'à propos du millénarisme. Non pas en termes d'influence, mais plutôt comme l'une des possibilités d'un schéma narratif. Ceux qui ont effectué l'itinéraire vers l'Autre Monde ont été modifiés intérieurement, et non pas seulement instruits, par cet itinéraire : ils vont maintenant mener une pieuse vie. Le narrateur utopique n'est qu'instruit; au plus étonné dans les premiers jours, admiratif dans les suivants. Mais rien qui lui dicte une conduite à tenir après son retour. Sauf celleci : raconter.

\section{NOTES}

1. Pierre Macherey, De l'utopie!, Strasbourg, De l'incidence éditeur, 2011, p. 37.

2. Karl Mannheim, Idéologie et utopie, Paris, Édition de la Maison de sciences de l'homme, 2006, p. 169.

3. Ibid., p. 168.

4. NDLR: Si la numérotation figure dans l'ouvrage original, les intertitres, placés entre crochets, ont été ajoutés par les éditeurs pour pouvoir faciliter la navigation en ligne. Les références ont elles aussi été mises, autant que faire se peut, aux normes de la revue, reprenant et complétant celles de l'original.

5. Raymond Trousson, Voyages aux pays de nulle part. Histoire littéraire de la pensée utopique, Bruxelles, Editions de l'Université de Bruxelles, 1975, p. 35. Ce continuisme n'empêche d'ailleurs pas l'ouvrage d'être un des meilleurs dont on dispose sur la question.

6. Thomas More, The Complete Works of St Thomas More, volume 4, ed. S. J. Edward Surtz et J. H. Hexter, Yale, Yale University Press, 1965, Y. 102, t. 97 [sic].

7. Raymond Trousson, Voyages aux pays de nulle part, op. cit., p. 33.

8. Cf. Victor Goldschmidt, Les dialogues de Platon, Paris, Puf, 1947, en particulier p. 1-14 et 274-305.

9. Victor Goldschmidt, Platonisme et pensée contemporaine, Paris, Aubier-Montaigne, 1970, p. 165-166.

10. Platon, Alcibiade, 129 c. II est vrai que l'anthropologie ne joue pas exactement le même rôle d'un dialogue à l'autre. Dans l'Alcibiade, la formule n'a qu'un sens négatif: l'identité n'est pas assurée par le corps. Dans la République, il y a au contraire une véritable construction de la notion d'âme.

11. Platon, Alcibiade, 432 a.

12. Par "éthique", il faut entendre ici ce qui concerne les relations entre les hommes. Le communisme dans la classe des gardiens sert ainsi à empêcher les sources de rivalité entre eux.

13. Aristote, Politique, Livre II.

14. Il faudrait comparer également l'utopie avec deux autres écrits de Platon, le Critias et les Lois. Alors que la République est un État conforme à la justice, et en principe réalisable (même s'il ne peut plus l'être), les Lois montrent un État proche du modèle juste, et réalisable en fait. Pas plus 
dans un cas que dans l'autre on ne trouve le schéma narratif qui suppose, pour la décrire, une société réalisée. Le système des Lois rentre dans la pratique grecque de la fondation de colonies (cf. Victor Goldschmidt, Platonisme et pensée contemporaine, op. cit., p. 166) et Léon Robin a montré il y a déjà longtemps à quel point elle essayait de prendre en compte les faits sociaux réels pour leur fournir des solutions adaptées et sûres (cf. Léon Robin, "Platon et la Science sociale ", in La Pensée hellénique. Des origines à Epicure, Paris, Puf, 1942, p. 177-230. Robin voyait dans les essais de réalisation tentés par Platon « un effet de cette conviction, profonde et sincère, que les faits ont été correctement observés, les types politiques classés, les éléments pathologiques, reconnus, les conditions acceptables d'une vie sociale normale bien déterminées »). En somme, les Lois rentrent dans la catégorie des projets d'instauration ou des programmes politiques, comme d'ailleurs un certain nombre d'œuvres traditionnellement classées dans l'Utopie : l'Oceana de Harrington, par exemple. Quant au Critias, qui avait pour dessein de raconter la guerre légendaire entre Athènes et les peuples de l'Atlantide, il décrit celle-ci comme une île où le dieu Poséidon avait installé les enfants qu'ils avaient engendrés d'une mortelle; si l'insularité fait penser au dispositif narratif repéré dans les récits utopiques, l'histoire en revanche nous en est racontée au passé, comme s'agissant de choses depuis longtemps disparues; nous en apprenons plus sur les généalogies des rois, la géographie physique de l'île et les cérémonies que sur l'organisation proprement politique et sociale. En fait, il n'y a rien là de proprement utopique : tout au plus des éléments extérieurs (forme de l'île, urbanisme) que des esprits formés aux lettres grecques pouvaient reprendre comme matériaux en constituant un genre nouveau. En tout cas on ne peut lire une continuité «utopisante» de la République au Critias : en effet, dans le second texte, c'est l'ancienne Athènes, et non l'Atlantide, qui est censée correspondre mythiquement à la société décrite dans le premier.

15. Hésiode, Les Travaux et les Jours, v. 109-126 (trad. P. Mazon, Paris, Les Belles-Lettres, 1928).

16. Ovide, Les métamorphoses, v. 89-93 (trad. G. Lafaye, Paris, Les Belles-Lettres, 1930).

17. Harry Levin, The Myth of the Golden Age in the Renaissance, Bloomington, Indiana University Press, 1969.

18. Ovide, Les métamorphoses, op. cit., v. 101-102.

19. Ibid., v. 107 (trait qui se retrouvera dans le Paradis de Milton).

20. Harry Levin, The Myth of the Golden Age in the Renaissance, op. cit., p. 89.

21. Cf. par exemple Lactance, Institutions divines, v. 5. (Le passage est cité par A. Bartlett Giammatti, The Earthly Paradise and the Renaissance Epic, Princeton, Princeton University Press, 1966, p. 31, en même temps qu'un texte de Prudence infiniment moins probant : Contre Symmaque, Livre II, vers 655 et suivants).

22. Harry Levin, The Myth of the Golden Age in the Renaissance, op. cit., p. XV.

23. Dante, Purgatoire, trad. H. Longnon, Paris, Garnier, 1966, chant XXVIII.

24. «Et le Seigneur Dieu prit l'homme qu'il avait créé et le plaça dans le Paradis pour le travailler et le garder " (Genèse, II, 15). Saint-Augustin commente ce texte dans le De Genesi ad litteram (VIII, $8,15)$ et répond à l'objection selon laquelle, puisque le travail est une punition (Gn, III, 17), il est peu vraisemblable que Dieu y ait déjà condamné le premier homme. « Nous le penserions, dit-il, si nous ne voyions certains s'adonner avec tant de plaisir au travail de la terre qu'ils ne l'abandonnent qu'à contre-cœur pour d'autres tâches. Quelque charme qu'ait d'ailleurs l'agriculture, elle en avait alors de bien plus grands, lorsque ni le sol ni le climat n'opposaient le moindre obstacle " (Saint-Augustin, De Genesi ad litteram, trad. P. Agaësse et A. Solignac, Paris, Desclée de Brouwer, 1972). On voit alors où passe la frontière : non pas entre l'inactivité qui serait le signe de la grâce et le travail, punition du péché, mais entre un travail joyeux et spontané (exhilaratio voluntatis) et le travail pénible (adflictio); la raison de cette différence résidant dans la malédiction jetée sur la terre - ce qui est strictement biblique. Le travail fait donc partie de la nature de l'homme : le Créateur lui avait donné l'art et la faculté de travailler, et la quantité qu'il fournissait de ce travail prenait sa mesure sur l'élan du cœur et non pas sur les 
besoins contraignants du corps. Il est clair qu'on pourrait transposer une telle distinction en Utopie : les utopiens cherchent un travail modéré, conforme à la raison, et (hors quelques uns, qui le font pour des raisons religieuses) ne sont pas des fanatiques du labeur pénible. S'ils détestent l'oisiveté, l'idéal qu'ils mettent en pratique est de repartir et d'organiser le travail de façon à le faciliter pour tout le monde. Évidemment, pour eux, il sert pourtant à satisfaire les besoins, et c'est de l'organisation sociale, non de la générosité d'une terre encore innocente qu'ils attendent cette modération. Par ailleurs il y a chez eux et chez leurs successeurs une volonté de maîtriser la nature que l'on chercherait en vain dans le commentaire augustinien. Mais on trouve une autre idée intéressante dans le même texte (VIII, 8, 16): le travail est formateur. En l'accomplissant, la raison humaine peut en quelque façon s'entretenir avec la nature: «L'homme, après avoir semé, planté les rejetons, transplanté les arbustes, greffé les boutures, interroge en quelque sorte la force vitale de chaque racine et de chaque germe, ce qu'elle peut ou ne peut pas, pourquoi elle le peut ou ne le peut pas, évalue ce que peut la puissance intérieure et invisible des nombres qui travaillent au-dedans, ce que peuvent les soins qu'on lui donne du dehors ». La connaissance ainsi acquise permet à l'homme de comprendre de façon plus systématique les différentes opérations de la Providence et de louer plus largement le Créateur. On retrouvera chez les utopistes, mais cette fois intégrée à un savoir plutôt orienté vers les techniques, cette conception d'un travail formateur de l'homme.

25. II faudrait, pour être complet, mentionner d'autres versions des Temps heureux, qui se distinguent elles aussi de l'utopie, bien qu'elles aient peut-être parfois servi de chaînons intermédiaires pour transmettre des thèmes - quitte à ce que ceux-ci soient repris dans une problématique leur conférant un sens différent. A) Les Champs Élysées, lieux de bonheur réservés aux héros grecs après leur mort (et distincts des Enfers, qui sont souterrains). Ainsi, au quatrième chant de l'Odyssée, Protée dit à Ménélas : "Aux Champs Élysées, tout au bout de la terre, chez le blond Rhadamanthe, où la plus douce vie est offerte aux humains, où sans neige, sans grand hiver, toujours sans pluie, on ne sent que zéphyrs, dont les risées sifflantes montent de l'Océan pour rafraîchir les hommes, les dieux t'emmèneront (Homère, L'odyssée, trad. V. Bérard, Paris, Gallimard, 1924, v. 565-9). B) Les îles Fortunées : territoires situés plus loin qu'aucun navigateur ne pourrait aller, et dont la légende a perduré pendant tout le Moyen-Âge, sous différentes formes; elles sont comme un fragment de l'Âge d'or qui aurait miraculeusement échappé à la décadence générale. Mais aussi bien que les Champs Élysées, elles ont de l'Âge d'or les traits négatifs : elles sont « à part » comme il était " pas encore »; elles n'ont aucune solution à suggérer aux problèmes de l'utopie, parce qu'elles ne peuvent tout simplement pas se les poser. Si ce n'est que leur statut d'îles peut suggérer un lien entre fermeture et bonheur : mais c'est une nostalgie, non une réflexion. C) Le pays de Cocagne : si lui aussi promet l'abondance (et même la surabondance et le gaspillage) c'est en partie par protestation et revanche contre la misère ; c'est «le paradis des gueux », comme le définit A.-L. Morton (Arthur Leslie Morton, L'utopie anglaise, Paris, Maspero, 1964, chapitre I). Il se présente en partie comme une compensation contre abus et injustices sociales, ce qui, certes, n'est pas sans faire penser au discours critique de l'utopie. Cependant les différences sont grandes: Cocagne est un pays impossible, qui satisfait spontanément les désirs en rêvant d'une inversion de la nature (le lait qui coule en rivière, les cochons qui s'offrent d'eux-mêmes à la fourchette); l'utopie est un monde possible, qui satisfait des désirs réglés par l'organisation sociale et la domination de la nature. A. Cioranescu explique cette démarcation en disant que Cocagne remplace un dieu par un autre (ou du moins par une Nature bienveillante et magique) alors que l'Utopie a conscience «que l'homme doit et peut se suffire à lui-même et que les dieux l'ont abandonné " (Alexandre Cioranescu, L'avenir du passé. Utopie et littérature, Paris, Gallimard, p. 60).

26. Gershom Scholem, Le Messianisme juif. Essais sur la spiritualité du judaïsme, Paris, Calman-Lévy, 1974, p. 29-31. 
27. On peut juger de ce rejet à l'occasion de l'évolution de Saint Augustin. Avant lui, la venue du règne $\mathrm{du}$ Christ sur la terre est censée se produire après les six âges du monde, pensés par analogie avec les six jours de la création : l'histoire est ainsi divisée en six «millénaires ", que suivra le Jour du Seigneur - c'est-à-dire le royaume de Dieu sur la terre qui durera à son tour mille ans ; il est évident qu'une telle doctrine, surtout lorsqu'on commence à calculer les temps et à prédire qu'ils sont bientôt venus, fait naître chez ceux qui y adhèrent une conscience de crise qui amène à dévaloriser les débris encore debout du sixième âge, destinés à être vite balayés. Saint Augustin, même lorsqu'il admettait la venue du Jour du Seigneur, s'était toujours refusé aux spéculations chiffrées. Il n'a donc pas été conduit à partager le pathos de l'Imminence que vivent tous les millénaristes. Au contraire, il a élaboré para la suite une seconde interprétation des âges du monde d'où disparaissait toute trace de millénarisme : Jésus est venu dès le sixième âge, celui dans lequel nous vivons encore (Saint Augustin, De Trinitate, IV, 4, 7) et qui durera jusqu'au terme inconnu du temps ; comme il s'était approché du puits à la sixième heure (Saint Augustin, Traités sur l'Evangile de Jean, XV, 8), il est descendu dans l'abîme de notre séjour ici-bas. Il n'y a donc plus de place pour l'attente d'une résolution soudaine de l'histoire. En revanche, chaque fois qu'en milieu chrétien on recommencera à spéculer sur les jours, les temps et les signes, la doctrine augustinienne sera écartée et on verra apparaître des prédictions appelant à se préparer pour le dernier jour, voire à le préparer.

28. Sur l'ensemble de ces tendances, voir, pour la Renaissance : Ernst Bloch, Thomas Münzer, théologien de la révolution, Paris, Julliard, 1962 ; Marianne Schaub, «Thomas Münzer : la nouvelle image de Dieu et le problème de la fin de l'histoire » in François Chatelet, Histoire de la philosophie, Paris, Hachette, 1972, t. III ; pour le XVII ${ }^{\mathrm{e}}$ siècle : Leszek Kolakowski, Chrétiens sans Église, Paris, Gallimard, 1969 ; Elisabeth Labrousse, "François Davant ou un millénariste au XVII ${ }^{\mathrm{e}}$ siècle ", in Centre d'histoire des sciences et des doctrines. Recherches sur le XVII ${ }^{e}$ siècle, I, Paris, Editions du CNRS, 1976, p. 73-86; et en particulier, sur le rôle joué par de telles doctrines dans la Révolution anglaise : Christopher Hill, Le Monde à l'Envers, Paris, Payot, 1977 ; et Olivier Lutaud, Cromwell, les Niveleurs et la République, Paris, Aubier, 1978.

29. Ainsi au XVII ${ }^{\mathrm{e}}$ siècle, en milieu juif, les variantes plus radicales donneront naissance au faux Messie Sabbataï Zvi (cf. Gershom Scholem, Le Messianisme juif, op. cit. et Gershom Scholem, Les grands courants de la mystique juive, Paris, Payot, 1977, p. 305-342); au contraire une réflexion plus modérée est celle de Menasseh ben Israël, qui par exemple refuse de se livrer à des spéculations numériques (cf. Menasseh ben Israël, Espérance d'Israël, Paris, Vrin, 1978).

30. Martin Buber, Utopie et Socialisme, Paris, Aubier, 1977 (avec une préface d'Emmanuel Lévinas), p. 26.

31. Gabriel de Foigny, La Terre australe connue, c'est-à-dire la description de ce pays inconnu jusqu'ici, de ses mœurs et de ses coutumes, Vannes (en fait, Genève) 1676 ; réédition dans Frédéric Lachèvre, Les successeurs de Cyrano de Bergerac, Paris, Champion, 1922, p. 61-163.

32. C'est pourquoi on ne peut approuver l'identification sommaire à laquelle procède Karl Mannheim, Idéologie et Utopie, trad. P. Rollet, Paris, Rivière, 1956 [NDLR : cette critique serait peut-être à nuancer en prenant en compte la traduction récente de cet ouvrage allemand, que nous citions en note 2. Pierre-François Moreau se réfère en effet à une traduction problématique en ce qu'elle a été effectuée non pas à partir de l'original allemand, mais à partir d'une traduction anglaise, et que ce texte anglais avait été lui-même dépouillé de la moitié de l'original]. Ce qui n'empêche pas que Mannheim analyse de façon admirable la conscience millénariste, en particulier ce qu'il appelle, chez Münzer, la présence absolue : «pour le vrai chiliaste, le présent devient la brèche par laquelle ce qui était auparavant intérieur jaillit soudain, s'empare du monde extérieur et le transforme » (op. cit., p. 160).

33. Jean Frappier, Du Moyen-Age à la Renaissance, études d'histoire et de critique littéraire, Paris, Champion, 1976, p. 24.

34. Jean Frappier, Histoires, Mythes et Symboles, Droz, Genève, 1976, p. 242. 
35. Jean Frappier, Du Moyen-Age à la Renaissance, op. cit., p. 25.

\section{AUTHOR}

\section{PIERRE-FRANÇOIS MOREAU}

Professeur de philosophie - ENS de Lyon. Directeur du laboratoire et responsable du CERPHI 